\title{
High-Grade Primary Osteosarcoma of the Thoracic Spine Presenting as an Ivory Vertebra
}

\section{Khin $Y^{1^{*}}$, Peh $W C G^{2}$, Chang $K T E^{3}$ and Mya $S^{4}$}

${ }^{1}$ Department of Diagnostic Radiology, Khoo Teck Puat Hospital, Republic of Singapore

${ }^{2}$ Senior Consultant, Clinical Professor and Head, Department of Diagnostic Radiology, Khoo Teck Puat Hospital, Republic of Singapore

${ }^{3}$ Department of Pathology and Laboratory Medicine, KK Women's and Children's Hospital, Republic of Singapore ${ }^{4}$ Department of Paediatric Oncology/Haematology, KK Women's and Children's Hospital, Republic of Singapore

*Corresponding author: Dr. Khin YT, Department of Diagnostic Radiology, Khoo Teck Puat Hospital, 90 Yishun Central, Singapore 768828, Republic of Singapore, Tel: (65)-6602-2689, Fax: (65)-6602-3796

\begin{abstract}
We report a 12-year-old Chinese girl with high-grade osteosarcoma of the thoracic spine, a rare site for a rare tumour. Radiograph showed a dense T9 vertebral body resembling an ivory vertebra. Bone scintigraphy revealed abnormal tracer uptake in the T9 vertebral body and posterior elements. Magnetic resonance imaging showed T1- and T2hypointense signal in the T9 vertebral body and the posterior elements, with diffuse enhancement. There was an associated heterogeneously-enhancing paravertebral soft tissue component surrounding the T9 vertebral body and the posterior elements, which extended into the epidural space encasing the thecal sac, spinal cord and bilateral intervertebral foramina. Computed tomography showed patchy but extensive mineralisation of all these soft tissue components. She was treated with T9 corpectomy, cord decompression and posterior instrumentation, followed by chemotherapy, with subsequent clinical improvement. The radiological features and differential diagnosis of thoracic spine osteosarcoma are discussed.
\end{abstract}

\section{Keywords}

Osteosarcoma, Primary spine tumour, Thoracic spine tumour

\section{Introduction}

Primary osteosarcoma of the spine is rare, accounting for $5 \%$ of all primary malignant tumours of the spine [1$3]$, and $0.4-2.7 \%$ of all osteosarcomas [1,4-6]. The most common sites of origin of this tumour are the metaphy- seal regions of the large tubular bones, although it may develop in any bone [2]. The ideal treatment is complete resection of the tumour and adjuvant chemotherapy [7]. Postoperative radiation therapy may be of benefit in selected patients [8]. We describe a young patient with a very aggressive osteosarcoma of the thoracic spine that presented innocuously as an ivory vertebra.

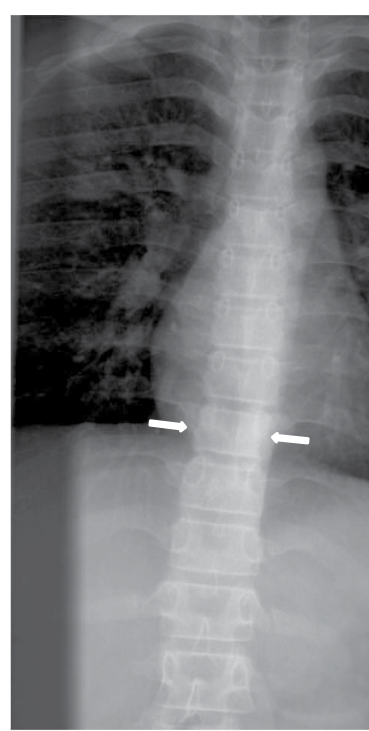

Fig 1a

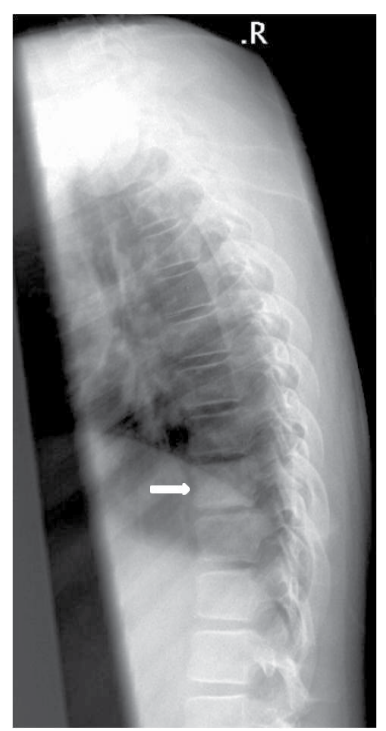

Fig 1b
Figure 1: Frontal and lateral radiographs show a mildly dense T9 vertebral body (arrows).

Citation: Khin YT, Peh WCG, Chang KTE, Mya SN (2018) High-Grade Primary Osteosarcoma of the Thoracic Spine Presenting as an Ivory Vertebra. Int J Cancer Clin Res 5:099. doi.org/10.23937/23783419/1410099

Accepted: October 25, 2018; Published: October 27, 2018

Copyright: (C) 2018 Khin YT, et al. This is an open-access article distributed under the terms of the Creative Commons Attribution License, which permits unrestricted use, distribution, and reproduction in any medium, provided the original author and source are credited. 


\section{Fig $2 a$}

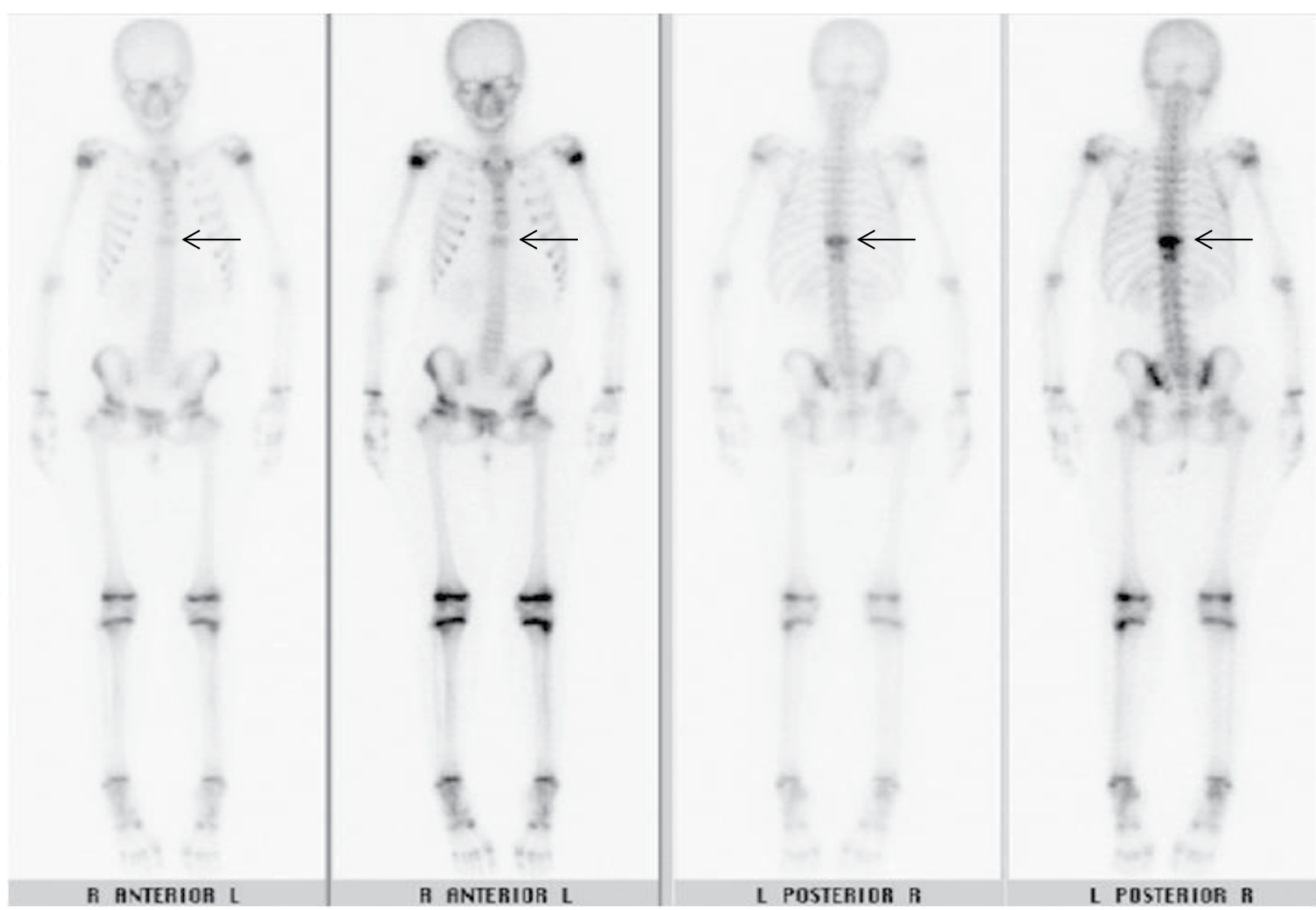

Fig $2 b$

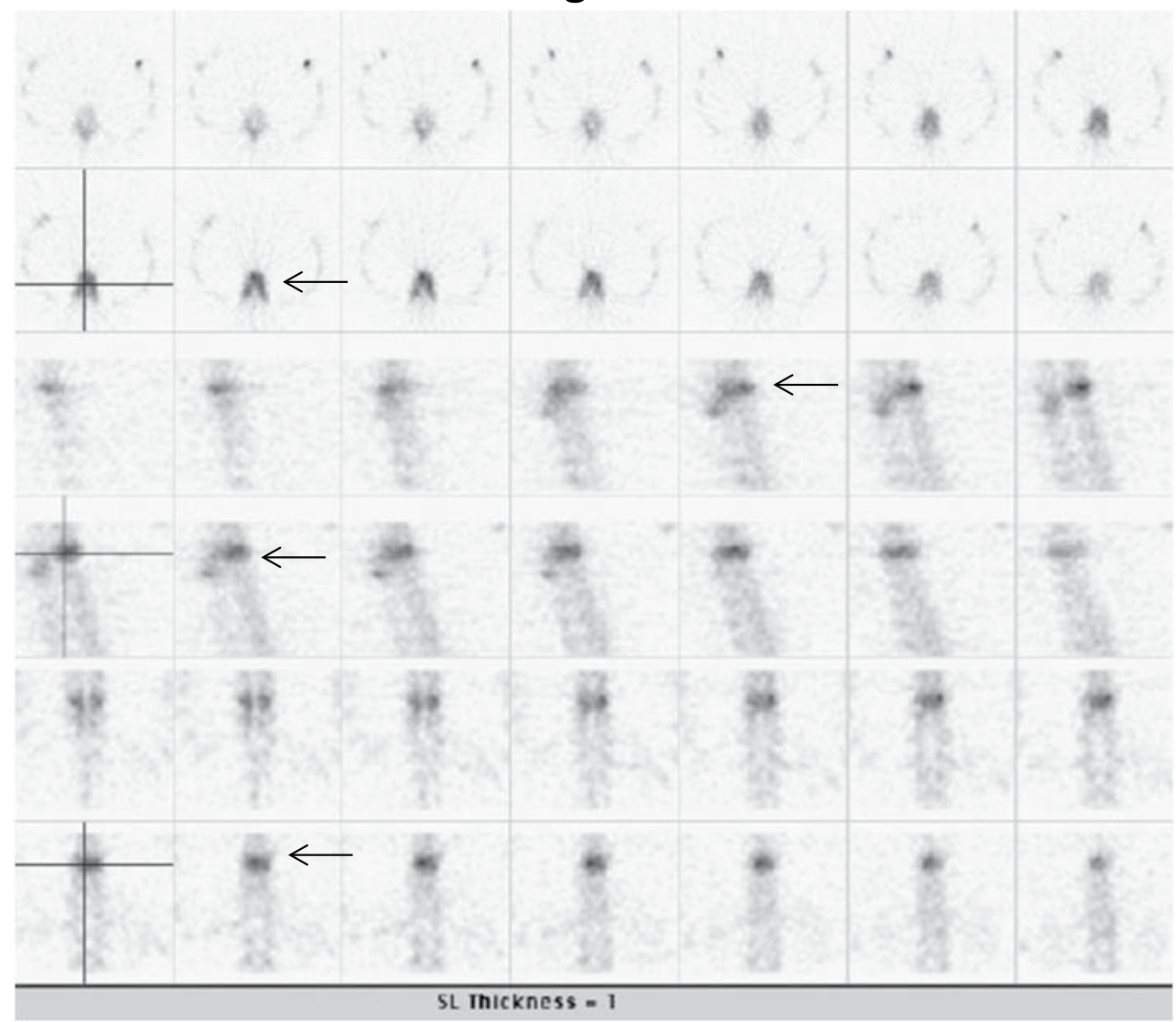

Figure 2: Tc99m-MDP (a) Delayed planar anterior and posterior and (b) SPECT bone scintiscans show intense focal tracer uptake in the T9 vertebral body and posterior elements (arrows). There was no other area of abnormal uptake in the rest of the skeleton. 


\section{Case Report}

A 12-year-old Chinese girl initially presented to the gastroenterology outpatient clinic in December 2009 with non-specific symptoms of epigastric pain radiating to the back for the past two to three months. Mild scoliosis was noted on the radiograph and the patient was referred to the Orthopaedic clinic. Review of the thoraco-lumbar spine radiograph showed mild thoracolumbar scoliosis and a mildly dense T9 vertebral body, resembling an "ivory vertebra". There was no loss of height or evidence of end-plate destruction. There was no definite soft tissue component, periosteal reaction or adjacent soft tissue mineralisation seen (Figure 1). The technetium-99m methylene diphosphonate (Tc99mMDP) bone scintiscan in December 2009 showed abnormal tracer uptake in the T9 vertebral body and posterior elements (Figure 2).

Magnetic resonance (MR) imaging of the thoraco-lumbar spine in January 2010 (Figure 3) showed abnormal signal in T9 vertebral body and the posterior elements, consisting of hypointense signal on T1-weighted and T2-weighted images and fat-saturated short T1 inversion recovery (FSTIR) images. These areas showed diffuse enhancement after intravenous Gadolinium-DTPA injection. There was an associated paravertebral soft tissue component surrounding the T9 vertebral body and the posterior elements, with heterogeneous enhancement. The abnormal soft tissue extended into the epidural space encasing the thecal sac and the spinal cord, causing a mass effect. The spinal cord had an ellipsoid appearance due to mass effect. The exiting nerve roots were not separately visualised as they were distorted by the soft tissue mass extending into both intervertebral foramina at the same level. Part of the paravertebral soft tissue extended to T8 and T10 vertebral levels, which was best seen in the coronal images (Figure 3). The height and endplates of the T9 vertebrae were preserved. There is no disc abnormality. The rest of the thoracic and the lumbar spine did not show any signal abnormality.

Based upon these MR imaging features, the differential diagnoses were osteosarcoma and lymphoma. Infective process was less likely as the end-plates and the adjacent discs were preserved and the patient did not have clinical signs of infection and the laboratory findings excluded infections such as bacterial, fungal and tuberculous infections. A computed tomography (CT)- guided biopsy of T9 vertebral body was performed on the same day as the MR Imaging but was not diagnostic. The patient remained neurologically well up to
Fig 3a

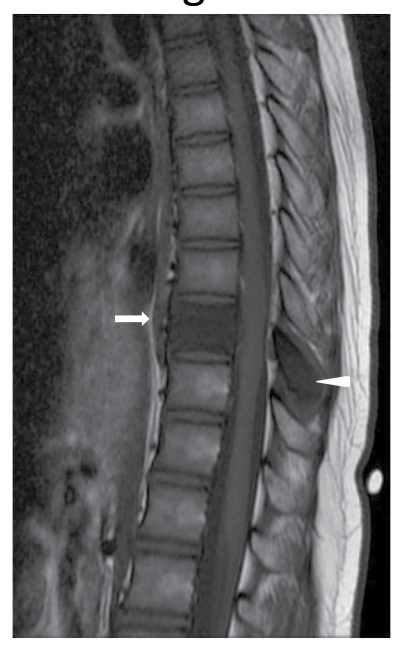

Fig 3d

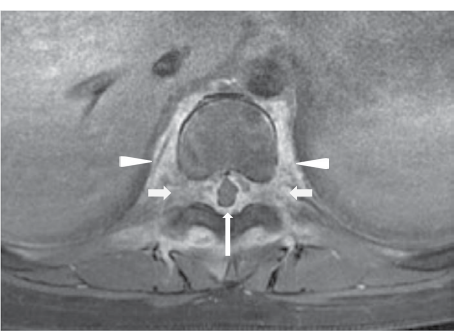

Fig 3b

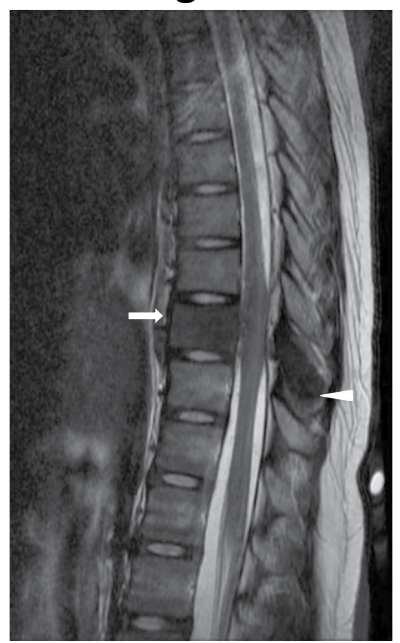

Fig 3c

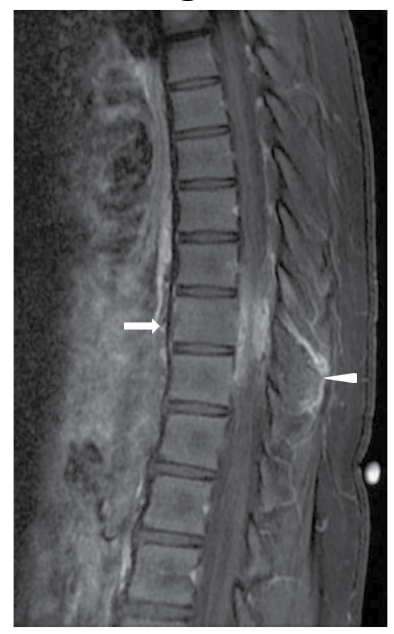

Fig $3 f$

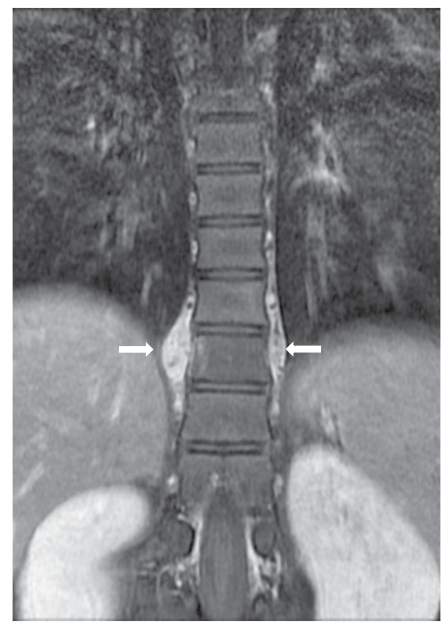

Fig 3e

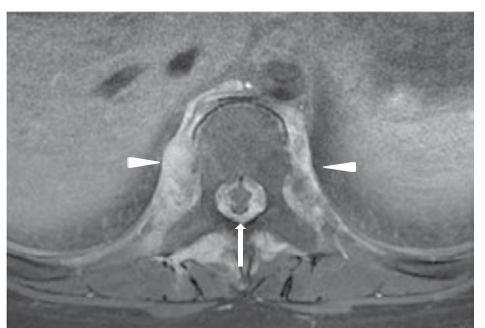

Figure 3: Sagittal (a) T1-W, (b) T2-W and (c) Post-contrast fat-saturated T1-W MR images show a hypointense T9 vertebral body (arrow) and spinous process (arrowhead), which enhanced diffusely. There is adjacent enhancing soft tissue which extends epidurally to compress the spinal cord. The adjacent intervertebral discs are unaffected. (d,e) Axial post-contrast fat-saturated T1-W MR images taken at T9 level better shows the diffusely-enhancing paravertebral soft tissue which extends around the vertebral body and posterior elements (arrowheads), and encases and compresses the spinal cord (thin long arrows), as well as both intervertebral foramina (thick short arrows). (f) Coronal post-contrast fat-saturated T1-W image shows the paraspinal soft tissue component extending from T8 to T10 vertebral levels (arrows). 


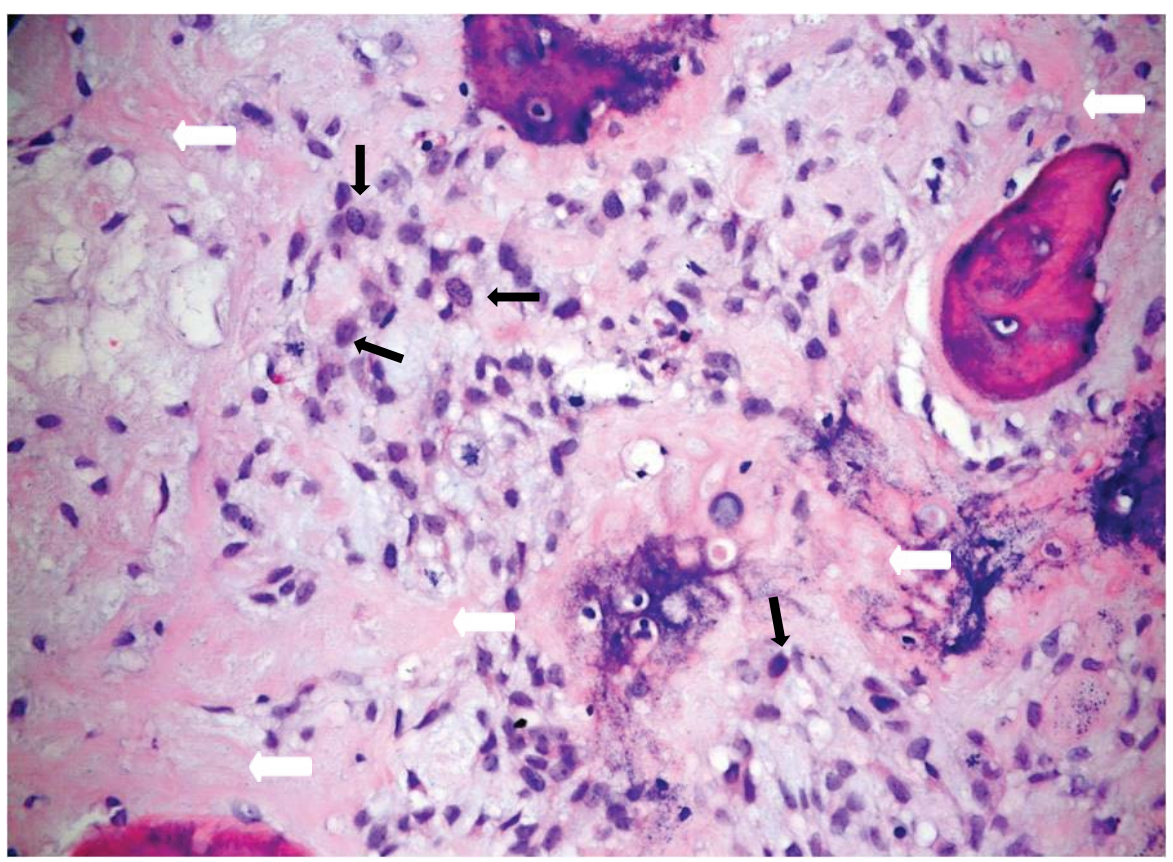

Figure 4: Photomicrograph of the T9 vertebral biopsy specimen shows atypical spindle cells (thin black arrows) producing osteoid (thick white arrows) with permeation into marrow spaces. Consistent with high-grade osteosarcoma (Haematoxylin \& eosin, $x 200)$.

\section{Fig $5 a$}

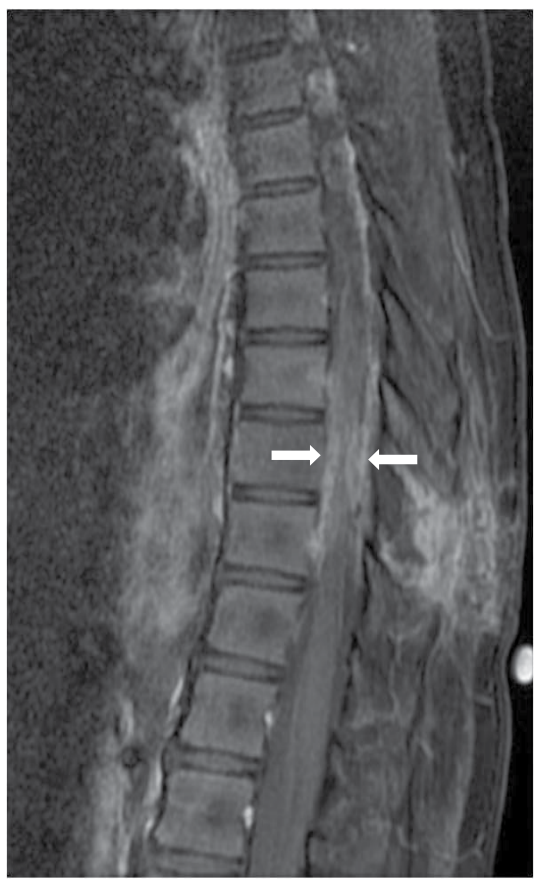

Fig $5 b$

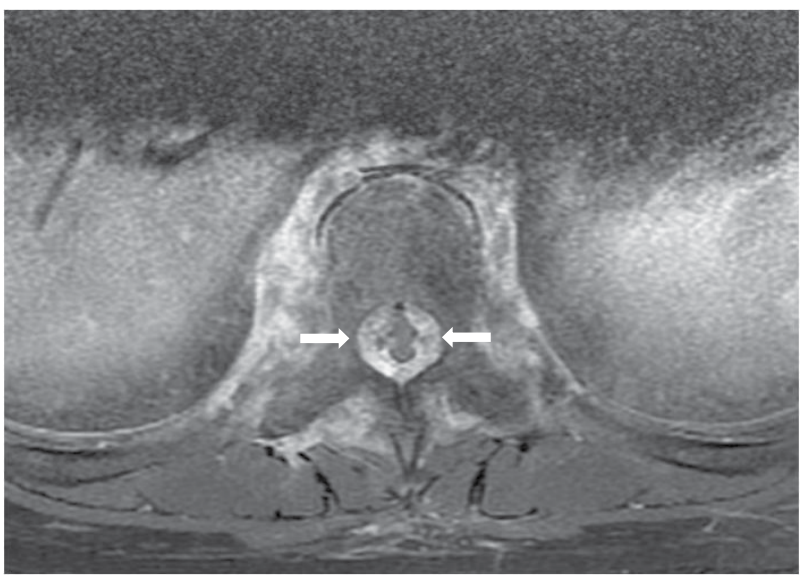

Figure 5: Repeat (a) Sagittal and (b) Axial post-contrast fat-saturated T1-W MR images show further progression of the soft tissue component, resulting in more severe compression of the spinal cord (arrows).

one week after the first MR imaging. The patient's gait then became more unsteady with occasional falls, and increasing weakness of the lower limbs. Open biopsy of T9 spinous process was performed 11 days later. This showed a tumour composed of atypical spindle cells which produced osteoid and which permeated the marrow spaces extensively, consistent with a high-grade osteosarcoma (Figure 4).
Six days after the biopsy, she developed significant sudden progressive worsening of bilateral lower limbs power, requiring support to walk, and one day of bowel incontinence. Urgent repeat MR imaging showed further mass effect on the thecal sac and the spinal cord by a soft tissue component, resulting in a slit-like appearance of the spinal cord (Figure 5). Computed tomography (CT) of the thorax performed later for staging did 
Fig $6 a$

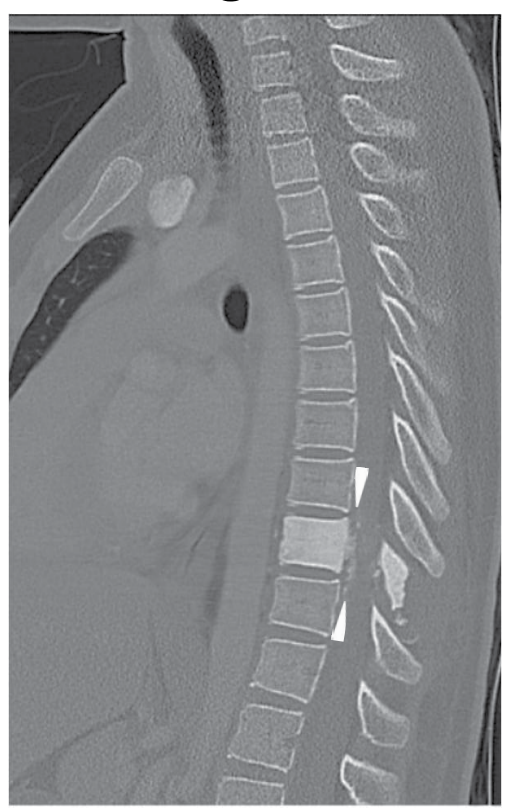

Fig 6b

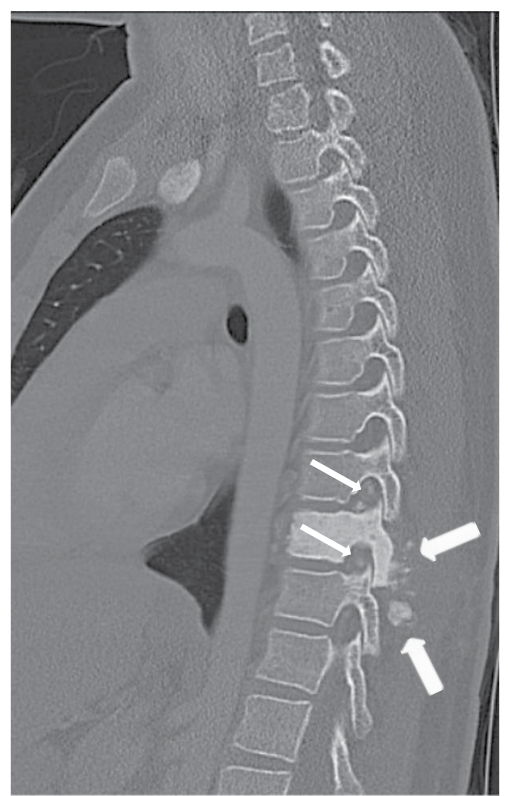

Fig 6c

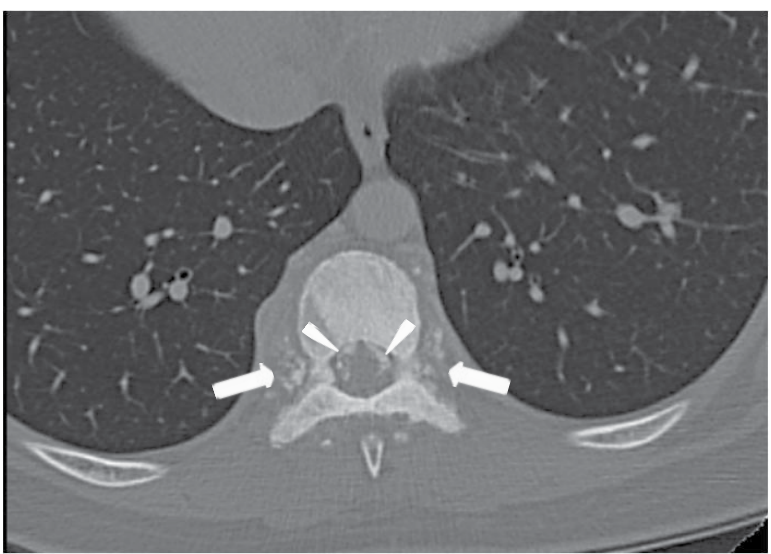

Figure 6: (a) Mid-sagittal, (b) Parasagittal and (c) Axial CT images show dense sclerosis of T9 vertebral body as well as of all the posterior elements (pedicles, laminae, transverse processes and spinous process). There is also patchy ossification of the paravertebral (thick arrows), epidural (arrowheads) and intervertebral foraminal (thin arrows) soft tissue components.

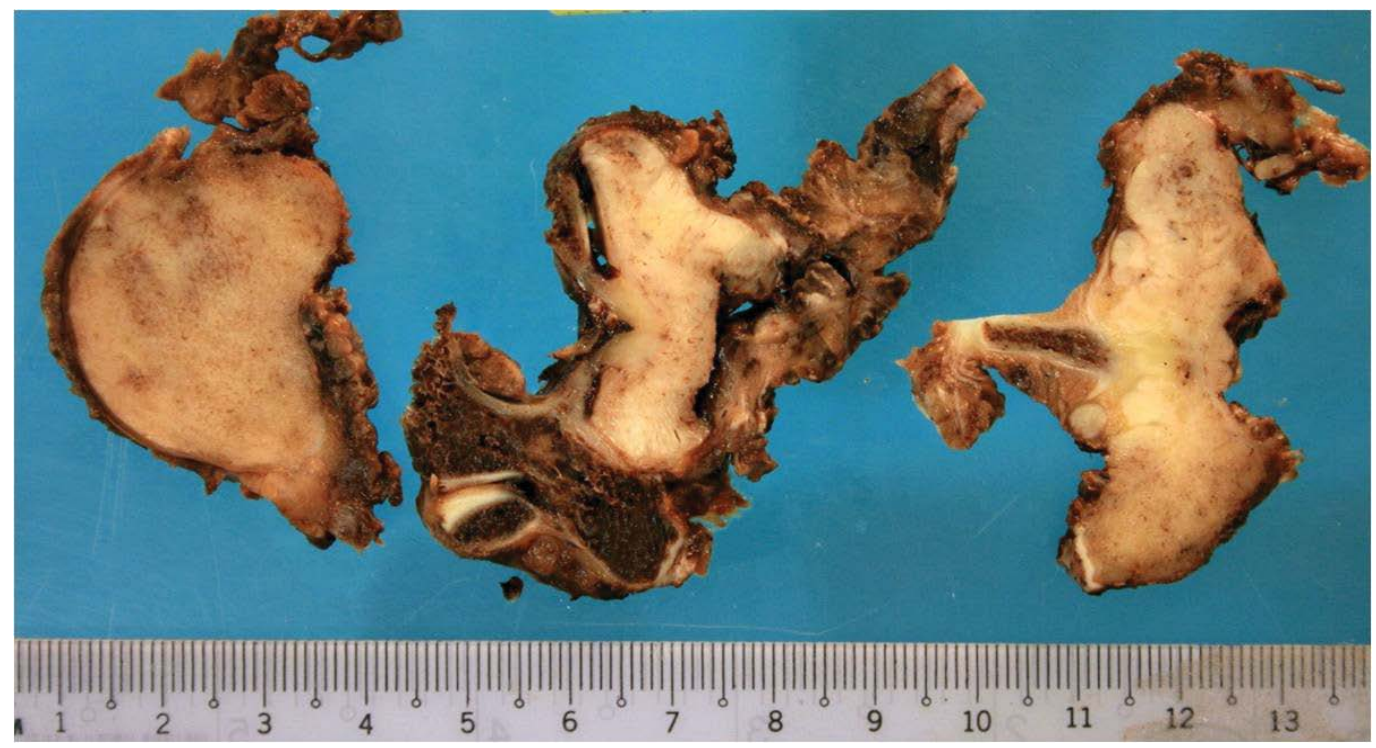

Figure 7: Photographs of the resected T9 corpectomy specimen show a white-grey appearance with firm consistency. The high-grade osteosarcoma was histologically similar to the previous biopsy specimen. 
not show evidence of pulmonary metastasis. On CT, the lesion consisted of sclerosis of the entire T9 vertebral body with patchy but extensive mineralisation of the surrounding soft tissue components including the paravertebral, epidural and foraminal soft tissues (Figure 6). These features were difficult to assess on MR imaging.

The patient underwent T9 corpectomy, cord decompression, and posterior instrumentation in January 2010. Intraoperatively, there was an infiltrative bony tumour, with an extensive soft tissue component involving the T8 to T10 posterior elements. Cord compression was seen at T9 level, with compression of the exiting nerve roots bilaterally. The resected specimen showed diffuse tumour infiltration of the vertebral body, posterior elements, costovertebral joints and pedicles, with a whitegrey gross appearance and firm consistency (Figure 7). The tumour was histologically similar to the open biopsy specimen. The tumour invaded into the surrounding fibroadipose and skeletal muscular tissues. There was also lymphovascular invasion and the tumour extended to the margins of the specimen. The patient was given chemotherapy according to Memorial Sloan-Kettering Cancer Centre, Non-Metastatic Osteosarcoma Protocol, consisting of high dose Methotrexate 12 doses, Doxorubicin 6 doses, Cisplatin 6 doses and Ifosfamide 3 doses. In total, 21 courses were administered from week 0 to week 38 (i.e. from 5 February 2010 to 15 November 2010).

MR imaging was repeated in May 2010 after induction chemotherapy (i.e. 2 cycles of Cisplatin, Doxorubicin, and 2 doses of Methotrexate (CDMM)). The posterior spinal instrumentation inserted from T7 to T11 caused artifacts, limiting the image clarity. There was moderate to severe spinal canal narrowing at the level of instrumentation (Figure 8). However, the previously-seen enhancing soft tissue mass compressing the spinal cord was no longer visualised. Except for post-operative changes, there was no evidence of tumour recurrence. Repeat CT of the thorax was also performed on the same day, which was normal without evidence of

\section{Fig 8a}

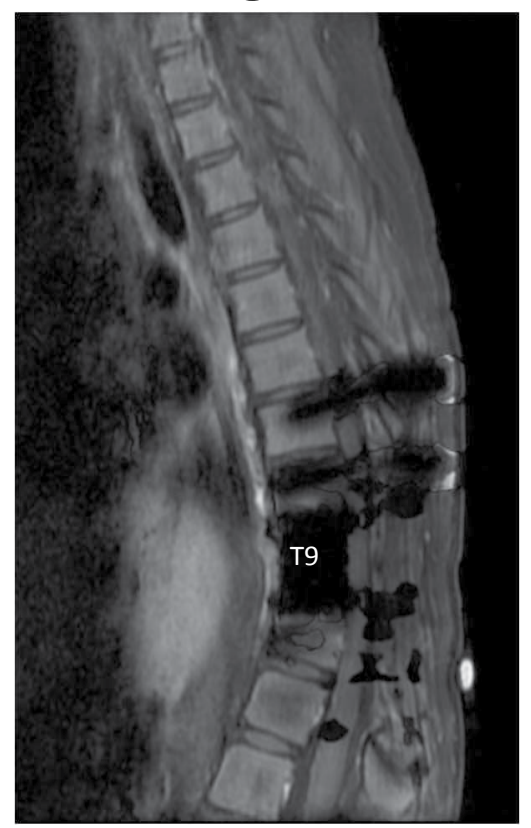

Fig $8 c$

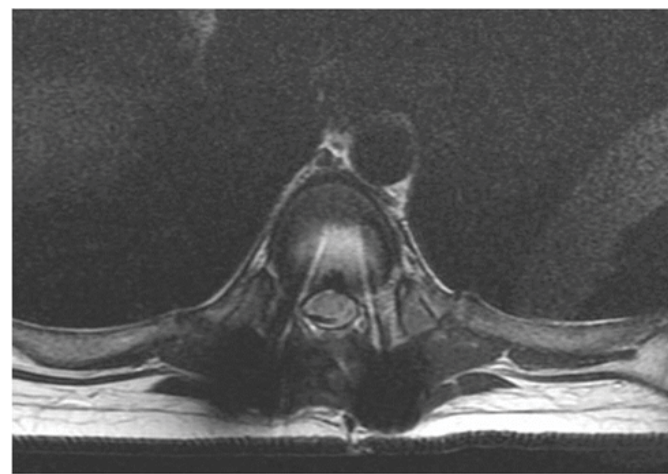

Fig $8 b$

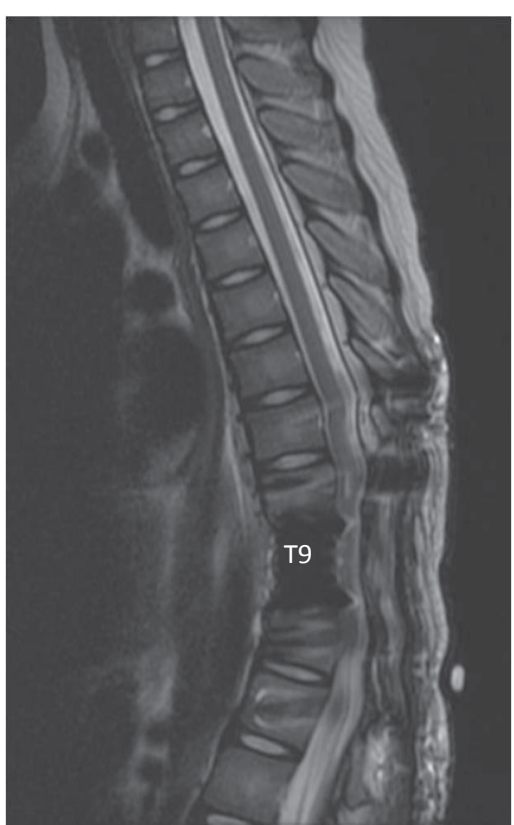

Fig 8d

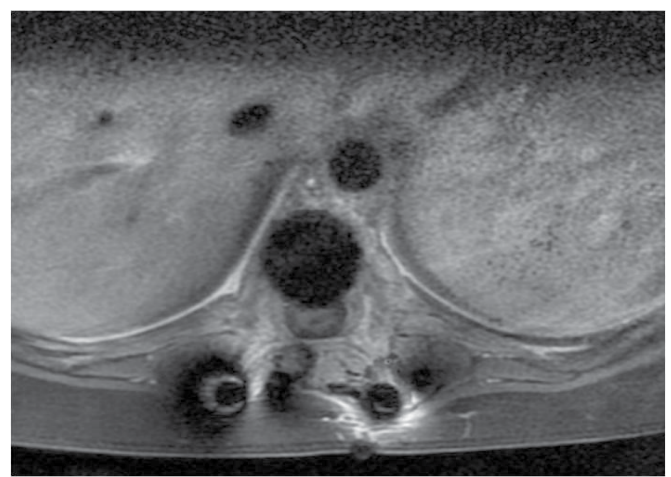

Figure 8: Post-chemotherapy repeat MR imaging. Sagittal (a) T1-W, (b) T2-W and axial (c) T2-W and (d) Post-contrast fat-saturated T1-W MR images show some artifacts due to spinal instrumentation. No evidence of mass lesion indicative of tumour recurrence. 
metastasis. Tc-99m MDP bone scintigraphy was repeated a few days later which showed a photopaenic area at the expected location of T9 vertebra due to previous surgery. There was no abnormal tracer uptake to suggest recurrence.

The patient completed all 21 courses of chemotherapy. After completion of chemotherapy, her condition improved clinically. She was able to walk with walking aids and was able to control her bowel functions. However, occasional inability to fully control her bladder still persisted. Follow-up MR imaging was performed up to December 2010 and CT of the thorax up to December 2011 did not reveal any suspicious lesion or recurrence.

She presented again with right-sided back pain for a week in August 2012 and repeated CT showed increase sclerosis in the entire T10 vertebral body which resembled the original tumour. There was however no pulmonary metastasis. Subsequent repeat MR imaging showed tumour recurrence at T10 vertebra with enhancing paravertebral soft tissue invading bilateral neural foramina at the same level and also extending cranially to T7-T8 vertebral levels. She underwent chemotherapy according to Relapsed or Progressive Osteosarcoma Protocol and radiotherapy to T6 to T12 vertebrae. Chemotherapy consisted of high dose Ifosfamide and Etoposide with Zoledronic acid (IEZ). She completed 5 cycles of IEZ and the last PET scan on 12 December 2012 showed significant reduction of hypermetabolic activity of T10 and T11 vertebral bodies with stable sclerosis, and no other hypermetabolic lesion seen elsewhere in the body.

\section{Discussion}

Osteosarcoma is a rare bone tumour but is still the most common primary malignant bone tumour seen in childhood. The most common sites of origin of this tumour are the metaphyseal regions of the large tubular bones, although it may develop in any bone [2]. Primary osteosarcoma of the spine is very uncommon, accounting for $0.4-2.7 \%$ of all osteosarcomas $[1,4-6]$. These tumours account for $5 \%$ of all primary malignant tumours of the spine [3]. There is no significant sex difference [9]. The age of the patients ranges from 8 to 80 years, with the median age of 34.5 years [2].

The patients often present with pain and a palpable mass, and $70 \%-80 \%$ have neurologic symptoms ranging from sensory deficits to paresis [1]. Serum alkaline phosphatase levels may be elevated. Osteosarcoma of the spine has been reported at all levels of the spine. The lumbosacral spine is the most common site, being reported in $60-70 \%$ of the patients [10]. Primary involvement of the posterior elements has been reported in $79 \%$, with partial vertebral body involvement [2].

Conventional radiographs are essential for evaluating a suspected bone lesion and should be obtained early in the work-up of symptomatic patients. It typically produces a densely mineralised matrix and may be seen as ivory vertebral body, with or without loss of vertebral height, and sparing of the adjacent disc [11]. Osteosarcoma, particularly when the posterior elements are involved, can be difficult to distinguish from osteoblasto$\mathrm{ma}$, both pathologically and radiologically $[1,12]$.

Tc-99m MDP bone scintigraphy is sensitive, but is not specific. However, multifocal lesions can be detected or excluded by this technique. CT and MR imaging are useful imaging modalities for evaluation of both the intraosseous extent of the tumour and soft tissue involvement. CT is the most accurate method in depicting the extent of osseous involvement, degree of cancellous and cortical bone loss, as well as any soft tissue mineralisation. MR imaging is the best imaging tool for evaluation of epidural space and neural structures, such as spinal cord and nerve root involvement. Lesions with a large amount of matrix mineralisation may remain of low signal intensity on all MR images, regardless of pulse sequence [11].

Our patient presented with subtle radiological features of an "ivory vertebra", or solitary vertebral sclerosis. In children, the finding of an ivory vertebra is less common and typically due to lymphoma, usually Hodgkin lymphoma. An ivory vertebra is less frequently due to osteosarcoma, metastatic neuroblastoma, medulloblastoma, osteoblastoma and more rarely, Ewing sarcoma. Osseous lymphoma often involves pedicles and destroys the posterior body cortex. An associated soft tissue mass is common with lymphoma. However, as shown in our case, the patchy but extensive mineralisation of the soft tissue component of the tumour narrows the diagnostic options to osteosarcoma. Ilaslan, et al. reported that the majority, 79\% (i.e. 55 out of 69 cases), of radiographs and CT of patients with vertebral osteosarcoma demonstrated mineralised matrix; and in 17 cases, marked mineralisation was observed. Five (7\%) of 69 cases with marked mineralisation were confined to the vertebral body, with a typical ivory vertebra appearance [2].

Metastasis may be focal and multiple, and may cause diffuse homogeneous marrow disease or occasionally, be seen as a solitary focal lesion. Osteosarcomas that arise in the spine have features identical to their appearances in any other bone, such as bone destruction, cortical erosion, periosteal new bone formation and soft tissue component which contains mineralisation. Rarely, osteosarcomas with marked mineralisation may manifest as an ivory vertebra, and may be known as sclerosing osteoblastic osteosarcoma [9]. Osteomyelitis is known to produce sclerosis in the vertebra during the healing phase; however, it rarely involves a single vertebra. Usually, erosive change is present at the margins of the intervertebral disc and this feature helps in the diagnosis [13].

The prognosis of spinal osteosarcoma is poor because the lesions are usually large at presentation and cannot be completely excised in this location. The treat- 
ment of the disease is resection of the tumour and adjuvant chemotherapy, as it is a relatively radioresistant malignancy. Radiotherapy is usually reserved for certain patients with unresectable tumours and/or where margins of resection are positive [14].

In summary, we report a patient with a high-grade primary osteosarcoma of the thoracic spine. Although primary osteosarcoma of the spine is rare, this diagnosis should be kept in mind when a large mineralised tumour involving the body and posterior elements of the spine is seen in a child with back pain. $\mathrm{CT}$ is very useful for showing mineralisation of the soft tissue components. Early detection, accurate diagnosis and appropriate treatment are crucial for prognosis, as well as for maintaining an adequate quality of life for the patient.

\section{References}

1. Barwick KW, Huvos AG, Smith J (1980) Primary osteogenic sarcoma of the vertebral column: a clinicopathologic correlation of ten patients. Cancer 46: 595-604.

2. Ilaslan H, Sundaram M, Unni KK, Shives TC (2004) Primary vertebral osteosarcoma: imaging findings. Radiology 230 : 697-702.

3. Green R, Saifuddin A, Cannon S (1996) Pictorial review: imaging of primary osteosarcoma of the spine. Clin Radiol 51: 325-329.

4. Bielack SS, Kempf-Bielack B, Delling G, Exner GU, Flege $S$, et al. (2002) Prognostic factors in high-grade osteosarcoma of the extremities or trunk: an analysis of 1,702 patients treated on neoadjuvant Cooperative Osteosarcoma Study Group protocols. J Clin Oncol 20: 776-790.
5. Ozaki T, Flege S, Liljenqvist U, Hillmann A, Delling G, et al. (2002) Osteosarcoma of the spine: experience of the Cooperative Osteosarcoma Study Group. Cancer 94: 1069-1077.

6. Brooks S, Starkie CM, Clarke NMP (1985) Osteosarcoma after the fourth decade. A clinico-pathological review. Arch Orthop Trauma Surg 104: 100-105.

7. Hogendoorn PC, ESMO/EUROBONET Working Group, Athanasou N, Bielack S, De Alava E, et al. (2010) Bone sarcomas: ESMO Clinical Practice Guidelines for diagnosis, treatment and follow-up. Ann Oncol 21: 204-213.

8. Murphey MD, wan Jaovisidha S, Temple HT, Gannon FH, Jelinek JS, et al. (2003) Telangiectatic osteosarcoma: radiologic-pathologic comparison. Radiology 229: 545-553.

9. Hsu CC, Lim KE, Hsu YY, Chan CY, Kuo HW, et al. (2007) Primary osteosarcoma of the spine: a case report. Chin J Radiol 32: 217-222.

10. Llauger J, Palmer J, Amores S, Bague S, Camins A (2000) Primary tumors of the sacrum: diagnostic imaging. Am J Roentgenol 174: 417-424.

11. Murphey MD, Andrews CL, Flemming DJ, Temple HT, Smith WS, et al. (1996) Primary tumors of the spine: radiologic-pathologic correlation. RadioGraphics 16: 1131-1158.

12. Kebudi R, Ayan I, Darendeliler E, Ağaoğlu L, Bayindir C, et al. (1994) Primary osteosarcoma of the cervical spine: a pediatric case report and review of the literature. Med Pediatr Oncol 23: 162-165.

13. Graham TS (2005) The ivory vertebra sign. Radiology 235: 614-615.

14. Federman N, Bernthal N, Eilber FC, William D (2009) The multidisciplinary management of osteosarcoma. Current Treatment Options Oncology 10: 82-93. 